\title{
Physical Statistics or Statistical Physics? A Brief Overview of Bayesian Melding
}

\author{
$\underline{\text { G.S. Chiu }}^{\text {a }}$, A.H. Westveld ${ }^{\text {bcd }}$ \\ ${ }^{\text {a } C S I R O ~ C o m p u t a t i o n a l ~ I n f o r m a t i c s, ~ G P O ~ B O X ~ 664, ~ C a n b e r r a, ~ A C T ~} 2601$ \\ ${ }^{\mathrm{b}}$ ESTeM, University of Canberra, Australia \\ ${ }^{\mathrm{c}}$ Statistics Laboratory @ Bio5 Institute, University of Arizona, USA \\ ${ }^{\mathrm{d}}$ Statistics Graduate Inter-disciplinary Program, University of Arizona, USA \\ Email: Grace.Chiu@csiro.au
}

\begin{abstract}
For scientists, a model is typically a set of mathematical formulae that describe some natural phenomena of interest. One approach, often preferred by applied mathematicians, engineers, and physicists, is to discover physical laws of nature and express them as deterministic mathematical relationships among quantities that comprise such laws. At the opposite end of the spectrum, physical laws that gave rise to the data may be of little concern to students in an undergraduate statistics course, whose emphasis is on discovering empirical relationships among observable quantities and construct regression models that relate both observable and unobservable quantities, including random noise. Practising statisticians express known physical laws in their regression models; what makes a regression model empirical is that the behaviour of deviations (noise) from the mathematical formulae is an integral part of the model itself. For years, Bayesian hierarchical modelling has been the statistical framework of choice to integrate empirical and deterministic modelling. Bayesian melding is a more recent alternative statistical framework: it expresses physical laws as laws, thus without explicit noise terms, yet it still allows the focus to be placed on the behaviour of random quantities. In this overview, we discuss some philosophical underpinnings of the Bayesian melding approach, and through a toy example we illustrate the nuances of formulating a Bayesian melding model. We list some published and ongoing research in ecology, economics, engineering, epidemiology, and population dynamics that employ Bayesian melding; these examples suggest the potential for Bayesian melding to unify deterministic and statistical modelling approaches in general.
\end{abstract}

Keywords: Bayesian melding, computer models, deterministic models, empirical models, hierarchical models, mathematical models, process models, simulation models, state-space models, statistical inference 


\section{INTRODUCTION}

Various modern scientific disciplines heavily involve interdisciplinary research by computer scientists, economists, engineers, mathematicians, physicists, and statisticians, among scientists in other fields; climate science is perhaps the poster child. While inherent differences among disciplinary philosophies can hinder scientific progress (Shaman et al., 2013), there is substantial common ground shared by these quantitative disciplines upon which joint effort can be built. Indeed, academic training in most of these disciplines invariably includes case studies in which 1) a set of mathematical formulae is devised to reflect some phenomenon, 2) data are being/have been collected and analyzed to justify the mathematical formulation, and 3) an implementation of this formulation is compared to the data in the context of calibration, or identification of what parts of the formulation require improvement. Step 1 is an integral part of modelling, whether the phenomenon be biological, chemical, physical, social, etc. Data are central to Step 2, an endeavour that is inherently statistical in nature. Step 3 ranges from very simple (e.g., calculating by hand a standard error according to some textbook formula and/or displaying observed data over model output then assessing the agreement visually) to highly complex (e.g., software engineers developing computer packages tailored to the specific model then domain scientists applying the software to produce model output for rigorous assessment of model adequacy with respect to observed data). In practice, Step 3 often feeds back into Step 1, and the whole process is iterated, constituting model refinement. Not only is this interdisciplinary approach already fundamental to many individual disciplines, it is employed typically early in a science education.

A physics student's world view. A simple example is the following experiment, similar to which many readers would have run in secondary school to determine the gravitational acceleration $g$ of a free falling object. In the experiment, the student releases a marble onto a floor mat from an adjustable platform fixed at each of 6 different heights between 0.5 and 3.5 metres, at $60-\mathrm{cm}$ increments. The platform and floor mat are connected through an electrical circuit that detects and displays the time elapsed from release to impact. The student enters the 6 readings on a computer spreadsheet to produce a plot resembling Fig. 1(a). The student is also instructed to transform the time recordings by squaring them, produce a new plot from the transformed data, run a linear regression through these data, then overlay the estimated regression line and associated confidence bands onto the plot, such as Fig. 1(b) with the red output. The final steps of the experiment are to estimate $g$ by inverting the slope estimate, and to apply a textbook formula to obtain the error bar range for $g$.

The purpose of transforming the data may seem obvious enough to the student: the very definition of acceleration relates distance to the square of time. Similarly, the regression analysis helps to determine the "best" estimate of $g$ based on the 6 observed data points. Yet, under the pressure of time, the student may scramble to carry out all steps as required by the experiment, let alone taking the time to fully appreciate the mysterious $R^{2}$ statistic, error bar for $g$, and confidence bands around the regression line. Perhaps a more pressing concern to the student is that the experiment was conducted correctly, in which case the estimate of $g$ should be close to the textbook value of 9.81 and the $R^{2}$ value, close to 1 .

Two statistics students' world views. In contrast, consider an introductory statistics course in which each student conducts an experiment with the same apparatus to empirically estimate the relationship between the height $(\mathrm{m})$ of free fall and time (s) spent in the fall, but in the absence of any instructions on data transformation. Upon seeing Fig. 1(a), Student A proceeds to fit a linear regression, feels satisfied that $R^{2}>0.98$, and reports the estimated relationship as "time $=0.186+0.123 \times$ height" with a p-value of 0.000. Unlike A, Student B regresses square-time on height, and produces the result in Fig. 1(b) in grey, also with $\mathrm{p}$-value $=0.000$. Both $\mathrm{A}$ and $\mathrm{B}$ are just as concerned as our physics student about running the experiment correctly, but what means do they have against which to gauge their empirical results? Realizing that their results differ drastically but class time is up, the two submit their reports in a cold sweat.

A realistic modeller's world view. Obviously, the above scenarios do not fully reflect the world views of practising physicists and statisticians. However, they shed light on the two ends of the spectrum of world view held by a quantitative modeller: 1) Mechanistic understanding informs the functional form that relates quantities of interest, and the objective is to determine the numerical values that specify the relation as a set of explicit formulae; data analyses are employed to justify the resulting formulae. 
(a)



(b)



Figure 1. (a) Time elapsed (s) plotted against fixed height (m) from a hypothetical free-fall experiment. (b) The same data after square-transformation of time; linear regression lines (solid) and 95\% confidence bands (dashed) are shown along with the estimated $x$-to- $y$ formulae, the $R^{2}$ statistics, and the estimated $g$ with error bar ranges: grey for regression with intercept and red, without intercept.

2) For descriptive and predictive purposes, the underlying but unknown functional form can be approximated purely empirically (based on data alone), and uncertainty in the approximated function and resulting predictions can be rigorously quantified; when uncertainty is deemed unacceptably large, alternative functional forms and/or additional data may be required. Such rigorous handling of uncertainty makes statistical modelling a powerful inference approach.

The realistic quantitative modeller's world view falls somewhere between 1) and 2) on the spectrum. In the case of the free-fall experiment, even if lacking a physics training, the modeller would insist on acquiring (from colleagues and/or literature reviews) a fundamental understanding of the response variable (time) and covariate (height). Then, not only would it be clear that the square-transformation is appropriate, but also that any linear regression should exclude an intercept. This way, the mechanistic understanding of a resting object is already embedded in the statistical model, and thus typical dangers of extrapolation into unobserved covariate ranges (Perrin, 1904) are mitigated.

Finally, the realistic modeller would ponder any limitations of simple linear regression as a tool to estimate $g$ from the free-fall data. Regression pertains to a statistical model. Here, the statistical model is

$(\text { observed time })^{2}=\beta \times($ observed height $)+$ error, error is iid Gaussian $\left(\right.$ mean $=0$, variance $\left.=\sigma^{2}\right)$

where "iid" denotes "independent and identically distributed." Thus, the model assumptions are i) "(time $)^{2} \propto$ height" represents the true state of nature, ii) time recordings are observed independently, the square of each has Gaussian noise, and iii) ignoring observation error in height, the Gaussian noise has a constant unknown variance $\sigma^{2}$.

Although it is common knowledge that i) is true, in a typical research scenario, how is the modeller to determine whether the presumed functional form is the true state of nature? If it is not, what does the error term represent, and how does that in turn affect the justification of assumptions ii) and iii)? After all, "garbage in, garbage out"-if linear regression were deemed unfit-for-purpose, then none of a high $R^{2}$, tight confidence bands, a small error bar range, or a small p-value would indicate a reliably estimated $g$.

Below, we explore these philosophical questions asked by the realistic quantitative modeller. We discuss these in the context of cutting-edge statistical modelling frameworks to encompass mechanistic and empirical modelling approaches; focus is on the Bayesian melding framework.

\section{TYPES OF ERROR}

"All models are wrong, but some are useful."

To fully appreciate this famous and seemingly obvious statement by the late statistician George E.P. Box, let us distinguish between the statistical (empirical) model (1) and its deterministic counterpart

$$
(\text { time })^{2}=\beta \times(\text { height }) \text {. }
$$

Effectively, the deterministic model (2) stipulates a presumed law of nature, without any reference to data. In this context, the wrongness or error in (2) would lie in that it may not be a universally true state 
of nature. (In the age of quantum mechanics, long-standing "universal" laws according to Newtonian physics indeed break down in the universe of subatomic particles.) This is model error.

Although (2) mentions no data, in practice the independent and dependent quantities in (2) are observable or measurable, thus referred to as variables by statisticians. In contrast, $\beta$ is a parameter that is not directly observable but can be "solved for" from the deterministic model given observations on the variables. However, observations or data are inherently noisy, so that each pair of (height, time) observation necessarily yields a unique "solution" for $\beta$ (ignoring rounding). This leads to the empirical version of (2), in the form of the equation in (1). Thus, the error term in (1) captures the randomness that violates the equality in (2). This is observation error.

In light of the model error in (2), can the error term in (1) be regarded merely as observation error? In practice, the answer is "no": an error term in a typical statistical model is a catch-all quantity that encompasses any deviation from the set of equalities that model the relationship among observed values. For example, the error term in (1) is, in practice, regarded as the overall wrongness of " (observed time $)^{2}=$ $\beta \times$ (observed height)" due to a possibly incorrect functional form and/or noisy observations.

Many statistical models fall in this category, from the simple regression model (1) to complex stochastic state-space models for nonlinear dynamical systems (e.g., McAllister et al., 1994; Parslow et al., 2013) that are often regarded as data assimilation frameworks - typically in the form of Bayesian hierarchical models - for rigorously integrating data and output from a deterministic model. However, the treatment in practice of the error terms in such models as catch-all can be at odds with the intended quantitative rigour that makes statistical inference a powerful tool. This is because inferential rigour relies on either that any intrinsic model error is negligible or that the inference procedure is robust to non-negligible model error, e.g., nonparametric methods which trade off statistical power for robustness. In most research scenarios, assuming negligible model error is unrealistic, although robustness of the inference procedure can be assessed analytically or through simulation studies.

To be or not to be Bayesian. Bayesian hierarchical models are multilevel models (Gelman and Hill, 2007) formulated under the Bayesian paradigm (Gelman et al., 2004)). A hierarchical model formulates the relationship among variables and parameters as a hierarchy of conditional dependence. For example, the two-level model

$$
y_{i}\left|w_{i}, \boldsymbol{\alpha} \sim p\left(y_{i} \mid w_{i}, \boldsymbol{\alpha}\right), \quad w_{i}\right| \boldsymbol{x}_{i}, \boldsymbol{\beta} \sim p\left(w_{i} \mid \boldsymbol{x}_{i}, \boldsymbol{\beta}\right), \quad i=1, \ldots, n
$$

expresses at Level 1 the probability distribution (generically denoted by $p$ ) of each data point $y_{i}$ conditioned on the knowledge of some random but possibly latent (not directly observable) quantity $w_{i}$ and parameter vector $\boldsymbol{\alpha}$, and at Level 2 the probability distribution of each $w_{i}$ conditioned on the knowledge of covariate vector $\boldsymbol{x}_{i}$ and parameter vector $\boldsymbol{\beta}$.

Many natural phenomena that are intrinsically hierarchical in nature can be formulated as hierarchical statistical models. Because statistical models can be formulated to formally integrate data and mechanistic understanding, hierarchical models are appealing stochastic counterparts of deterministic models to reflect complex processes. Hierarchical models need not be Bayesian, however, e.g., de Valpine (2012) considers state-space models in the frequentist framework under which parameters are regarded as nonrandom. For complex models, one advantage of the Bayesian approach is that plausible ranges of unknown parameters can be expressed explicitly as a model component in the form of prior probability distributions. For example, a modeller for (1) may specify the prior $\beta^{-1} \sim \operatorname{Gaussian}(10,1)$ to reflect the $a$ priori understanding (outside of the current experiment) that free falling objects near the surface of the earth exhibit a typical acceleration of around $10 \mathrm{~ms}^{-2}$, give or take 1 . In addition to observed data, prior distributions contribute auxiliary information about the phenomenon at hand, thus they can improve statistical power and hence, reduce estimation uncertainty. Priors can be specified at virtually any level of informativeness, with tighter distributions being more informative. Another advantage is the flexibility in a Bayesian model's functional form and the types of probability distributions involved to accommodate nonstandard estimation problems, e.g., those without closed-form solutions. This is in contrast to many frequentist approaches that rely on closed-form asymptotic theory that only applies when the functional form and data collection process satisfy the regularity conditions required for the theory to be valid. Note that not requiring closed-form solutions typically implies computational burden (e.g., Murray, 2013). 


\section{BAYESIAN MELDING: A DIFFERENT PERSPECTIVE OF ERROR}

Deterministic models are also known as computer models, mathematical models, or simulation models. Process models are such models formulated to reflect complex processes. Deterministic models typically involve so-called input and output quantities. Output quantities are of main interest; they are solved for given input quantities. In the case of (2), time and height are input quantities and $\beta$ is the output quantity.

To understand bowhead whale population dynamics, Raftery et al. (1995) proposed the statistical modelling framework Bayesian synthesis which was subsequently refined by Poole and Raftery (2000) into the Bayesian melding framework. Their application involved a standard population dynamics model but no explicit catch-all error term. Instead, the set of equations that constitute a deterministic model is kept intact under Bayesian melding, and stochasticity is expressed hierarchically through prior distributions and likelihood functions (probability distributions for data) associated with quantities in the equations. The novelty of the approach is that each model parameter that would have been solved for in the context of deterministic modelling would be associated with an induced prior in addition to a pre-(deterministic-)model prior. While Bayesian melding is an obvious overkill for modelling free fall, as a toy example, a Bayesian melding formulation that corresponds to (2) is still nontrivial, comprising the four main components of

likelihood: $\quad t_{i} \mid \gamma_{0}, \gamma_{1}, h_{i} \sim \operatorname{Gaussian}\left(\gamma_{0}+\gamma_{1} h_{i}, 0.05^{2}\right)$,

$h_{i}$ are nonrandom covariate values,

$$
\begin{aligned}
\text { deterministic model: } & \beta=M\left(\gamma_{0}, \gamma_{1}, \boldsymbol{h}\right) \quad \text { where } \quad M\left(\gamma_{0}, \gamma_{1}, \boldsymbol{h}\right)=\frac{1}{6} \sum_{i=1}^{6} \frac{\left(\gamma_{0}+\gamma_{1} h_{i}\right)^{2}}{h_{i}}, \\
\text { premodel priors: } & \gamma_{0}, \gamma_{1} \sim \text { iid uniform }(0,0.5), \quad \beta^{-1} \sim \operatorname{Gaussian}(10,1), \\
\text { induced prior: } & \beta \sim p^{*}(\beta \mid M, \boldsymbol{\gamma}, \boldsymbol{h})
\end{aligned}
$$

where $\left(h_{i}, t_{i}\right)$ denotes the height and time of the $i$ th marble drop. The likelihood is based on assuming a linear empirical relationship between height and time-as would be a reasonable specification by our statistics Student A above-and a standard deviation of $0.05 \mathrm{~s}$ for time given height, which may be deduced from the operations manual for the electronic sensor. The deterministic model $M$ is what the modeller wishes to incorporate with data (the likelihood). Thus, in the context of the modeller's freefall experiment and likelihood, (time $)^{2}$ would correspond to $\left(\gamma_{0}+\gamma_{1} \times\right.$ (height $\left.)\right)^{2}$; the summation in $M$ is to reconcile the theoretically constant value for $\beta$ with the 6 different "solutions" for $\beta$ from (2). The premodel priors reflect a priori understanding (outside of the experiment and of $M$ ) about the input parameters $\gamma_{0}$ and $\gamma_{1}$ and output parameter $\beta$. Now, consider feeding $\boldsymbol{h}$ and $p_{\text {premodel }}\left(\gamma_{0}, \gamma_{1}\right)$ through the deterministic model $M$. This incurs or induces a probability distribution for $\beta$, which is $p^{*}(\beta \mid M, \gamma, \boldsymbol{h})$.

Statistical inference is done in the Bayesian paradigm, such that likelihood and prior distributions are integrated to form the joint posterior distribution for model parameters. Because two sets of prior distributions exist for output quantities, they must be melded to produce a single melded output prior. The logarithmic pooling method with pooling weight $\alpha$ yields the melded output prior

$$
p_{\text {melded }}(\beta) \propto\left[p^{*}(\beta)\right]^{\alpha}\left[p_{\text {premodel }}(\beta)\right]^{1-\alpha}, \quad \alpha \in[0,1] .
$$

This allows the modeller to specify the tuning parameter $\alpha$ to reflect how much the statistical inference should rely on $M$. For example, by fixing $\alpha=0$, the deterministic model $M$ would be ignored altogether and standard Bayesian inference would proceed. At the other extreme, the modeller could discredit any a priori understanding about $\beta$, thus letting $M$ fully dictate the pre-inference behaviour of $\beta$.

Given $p_{\text {melded }}(\beta)$, the model $M$ in turn constrains the behaviour of $\gamma$. In principle, feeding $p_{\text {melded }}(\beta)$ through $M^{-1}$ would lead to a joint melded input prior $p_{\text {melded }}(\gamma)$, although $M$ is obviously uninvertible. Poole and Raftery (2000) proved that logarithmic pooling ensures regularity even for an uninvertible $M$. In particular, $p_{\text {melded }}(\gamma)$ is not explicitly involved in the Bayesian inference procedure, but inference is instead based on the melded input posterior distribution

$$
\pi_{\text {melded }}(\gamma) \propto p_{\text {premodel }}(\gamma)\left[\frac{p_{\text {premodel }}(\beta=M(\gamma))}{p^{*}(\beta=M(\gamma))}\right]^{1-\alpha}
$$

as derived by Poole and Raftery (2000) for logarithmic pooling. Finally, feeding $\pi_{\text {melded }}(\gamma)$ through $M$ 

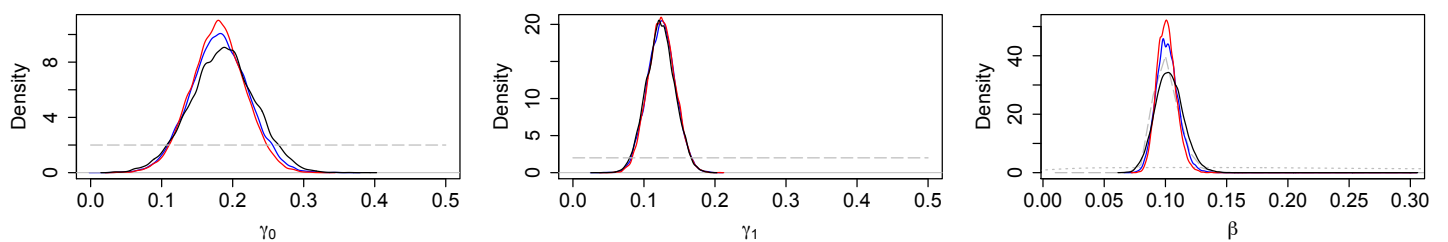

Figure 2. Inference for $\gamma_{0}, \gamma_{1}$, and $\beta$ based on our Bayesian melding formulation of the free fall experiment. Solid curves are melded posteriors (red: $\alpha=0.05$; blue: $\alpha=0.5$; black: $\alpha=0.995$ ). Broken curves denote priors (long dashes: premodel; short dashes: induced).

gives the melded output posterior $\pi_{\text {melded }}(\beta)$. Fig. 2 presents the melded input and output posteriors.

As is the case for all modelling exercises, the same scientific problem may be formulated for Bayesian melding differently through various combinations of output parameters, likelihood, and $M$. But the same nuances are universal, even for our seemingly trivial toy example: to ensure that all four components of Bayesian melding are sensibly defined (e.g., what constitutes an output as opposed to an input parameter) and structured to contribute to statistical inference along an appropriate inference pathway. Here, our formulation is such that $\beta$ is the output parameter, and that the informational content of the data $\{\boldsymbol{h}, \boldsymbol{t}\}$ bubbles up to $\beta$ through the input $\gamma$ and the model $M$, then the inference for $\beta$ is fed back to the inference of $\gamma$ through $M$ (in the opposite direction), thus completing the feedback loop of the inference pathway.

\section{SOME SCIENTIFIC APPLICATIONS OF BAYESIAN MELDING}

Since the seminal study by Poole and Raftery (2000) on whale population dynamics, several scientific applications of Bayesian melding have appeared in the literature, all of which illustrate the appeal of the approach for integrating data modelling with deterministic modelling. For example, Chiu and Gould (2010) considered a simple mass balance model for nutrient transfer in a 36-compartment food web ecosystem: $\phi=\theta_{1}-\theta_{2}$, where $\phi, \theta_{1}$, and $\theta_{2}$ respectively denote the mean dissipation, mean influx, and mean outflux, each being the average across all 36 compartments. The output parameter $\phi$ and input parameters $\theta_{1}$ and $\theta_{2}$ each have a premodel prior and likelihood, the latter coming from data on dissipation, influx, and outflux. Chiu (2012) presented a minimally technical summary of this work. Other ecological applications of Bayesian melding have concerned forest systems (Radtke et al., 2002; Radtke and Robinson, 2006). Applications for soil erosion mechanism (Falk et al., 2009) and disease transmission (Spear et al., 2002) also have emerged in the literature. Currently, the authors of this overview are leading research that develops specialized models that employ Bayesian melding respectively in the engineering sciences and economics. Other CSIRO scientists are currently pursuing Bayesian melding in disease detection. Meanwhile, after the seminal methodological paper, Raftery continued to collaborate on Bayesian melding applications in diverse disciplines including epidemiology (Alkema et al., 2007, 2008) and air quality control (Fuentes and Raftery, 2005).

\section{Conclusion}

Stochastic state-space modelling virtually has been the default data assimilation framework in quantitative sciences, partly due to the well-established body of methodological and applied research associated with it. For example, the widely used Kalman filter falls under this framework (Brockwell and Davis, 2006). Nonetheless, since it first appeared in the literature in 2000, Bayesian melding has been an appealing alternative that allows the modeller to take a perspective of model and data errors that is different from what pertains to conventional statistical modelling frameworks.

Staying true to the philosophy of deterministic modelling, Bayesian melding regards a deterministic model $M$ as the presumed law of nature. To reconcile $M$ with data which necessarily violate the equalities as stipulated by $M$, likelihood functions can be specified for those model parameters that are associated with these data. A priori understanding of the model parameters can also be specified as premodel prior distributions. These specifications are constrained through $M$ which maps the input parameters to output parameters, and conversely from output to input, even if $M$ is uninvertible. The Bayesian melding framework is flexible in that the modeller can tune the inference according to a preconceived level of reliability 
for the deterministic model relative to the a priori understanding of the unknown quantities of interest. In principle, the tuning parameter $\alpha$ (logarithmic pooling weight) can even be estimated empirically if it is made a model parameter under the Bayesian melding modelling framework.

\section{ACKNOWLEDGEMENT}

GC thanks J Taylor (CSIRO Computational Informatics; CSIRO eResearch; CSIRO Computational and Simulation Sciences) for his moral support of research involving Bayesian melding and his financial support through sponsoring GC's involvement in CSIRO Computational and Simulation Sciences. We thank B Robson and the referees for their valuable suggestions that helped to improve this article.

\section{REFERENCES}

Alkema, L., A. E. Raftery, and T. Brown (2008). Bayesian melding for estimating uncertainty in national HIV prevalence estimates. Sexually Transmitted Infections 84, i11-i16.

Alkema, L., A. E. Raftery, and S. J. Clark (2007). Probabilistic projections of HIV prevalence using Bayesian melding. The Annals of Applied Statistics 1, 229-248.

Brockwell, P. J. and R. A. Davis (2006). Time Series: Theory and Methods (2 ed.). New York: Springer.

Chiu, G. S. (2012). Food web modeling. In A. H. El-Shaarawi and W. W. Piegorsch (Eds.), Encyclopedia of Environmetrics (2 ed.)., Chichester, pp. 1063-1066. Wiley.

Chiu, G. S. and J. M. Gould (2010). Statistical inference for food webs with emphasis on ecological networks via Bayesian melding. Environmetrics 21, 728-740.

de Valpine, P. (2012). Frequentist analysis of hierarchical models for population dynamics and demographic data. Journal of Ornithology 152, S393-S408.

Falk, M. G., R. J. Denham, and K. L. Mengersen (2009). Estimating uncertainty in the revised universal soil loss equation via Bayesian melding. Journal of Agricultural, Biological, and Environmental Statistics 15, 2006.

Fuentes, M. and A. E. Raftery (2005). Model evaluation and spatial interpolation by Bayesian combination of observations with outputs from numerical models. Biometrics 61, 36-45.

Gelman, A., J. B. Carlin, H. S. Stern, and D. B. Rubin (2004). Bayesian Data Analysis (2 ed.). Boca Raton: Chapman and Hall/CRC.

Gelman, A. and J. Hill (2007). Data Analysis Using Regression and Multilevel/Hierarchical Models. New York: Cambridge University Press.

McAllister, M. K., E. K. Pikitch, A. E. Punt, and R. Hilborn (1994). A Bayesian approach to stock assessment and harvest decisions using the sampling/importance resampling algorithm. Canadian Journal of Fisheries and Aquatic Sciences 51, 2673-2687.

Murray, L. M. (2013). Bayesian state-space modelling on high-performance hardware using LibBi. arXiv, $1306.3277 \mathrm{v} 1$.

Parslow, J., N. Cressie, E. P. Campbell, E. Jones, and L. Murray (2013). Bayesian learning and predictability in a stochastic nonlinear dynamical model. Ecological Applications 23, 679-698.

Perrin, E. (1904). On some dangers of extrapolation. Biometrika 3, 99-103.

Poole, D. and A. E. Raftery (2000). Inference for deterministic simulation models: the Bayesian melding approach. Journal of the American Statistical Association 95, 1244-1255.

Radtke, P. J., T. E. Burk, and P. V. Bolstad (2002). Bayesian melding of a forest ecosystem model with correlated inputs. Forest Science 48, 2002.

Radtke, P. J. and A. P. Robinson (2006). A Bayesian strategy for combining predictions from empirical and process-based models. Ecological Modelling 190, 2002.

Raftery, A. E., G. H. Givens, and J. E. Zeh (1995). Inference from a deterministic population dynamics model for bowhead whales. Journal of the American Statistical Association 90, 402-430.

Shaman, J., S. Solomon, R. R. Colwell, and C. B. Field (2013). Fostering advances in interdisciplinary climate science. Proceedings of the National Academy of Sciences of the USA 110, 3653-3656.

Spear, R. C., A. Hubbard, S. Liang, and E. Seto (2002). Disease transmission models for public health decision making: Toward an approach for designing intervention strategies for Schistosomiasis japonica. Environmental Health Perspectives 110, 2002. 